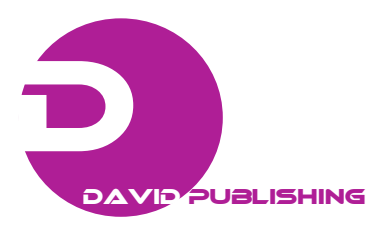

\title{
Korean Perspectives on Food Safety and Regulations
}

\author{
Deog-Hwan Oh \\ Department of Food Science and Biotechnology and Institute of Bioscience and Biotechnology, Kangwon National University, \\ Chuncheon, Gangwon 200-701, Republic of Korea \\ Corresponding author's e-mail: deoghwa@kangwon.ac.kr
}

\begin{abstract}
Recently, food safety has become extremely important and ensuring products safety is an international public health concern as well as in Korea. Despite the prominent advances in the Food Science Technology, there is still further necessity of improving food hygiene technologies due to climate change, emergence of new type food and change of social dietary patterns. Since Korea food import has been increasing every year it is quite necessary to establish sufficient regulations and actively update them to ensure consumers safety especially from the global food issues. Therefore, the conventional actions taken in Korea in 2008 based on initial sample examination, provisional prohibition of sales/marketing and legal actions against contaminated products to manage melamine incident in China were replaced with the advanced "smart ecosystem" technology to minimize the consequences of Japan 2011 nuclear crisis on Korean consumers health. Smart ecosystem is an integrated technology based on real-time information that excludes problems such as repeated collection of data, poor data reliability, lack of real-time information and burden of paper work in the conventional food safety management. Utilization of real-time information, saving business process time, improvement of data reliability and establishment of real-time situation management are among the advantages of the newly developed smart ecosystem upon the conventional approaches. These advantages make this system a great tool for nationwide surveillance and monitoring. Thus, a strengthened international smart ecosystem is necessary for the rapid safety information sharing through global cooperation. Such collaborative efforts may significantly improve food safety and consumers health in an international scale.
\end{abstract}

Key words: Food safety, global food issues, smart ecosystem, consumers’ health. 